\title{
Ghosal hematodiaphyseal dysplasia
}

INSERM

\section{Source}

INSERM. (1999). Orphanet: an online rare disease and orphan drug data base. Ghosal hematodiaphyseal dysplasia. ORPHA:1802

Ghosal hematodiaphyseal dysplasia syndrome (GHDD) is a rare disorder characterized by increased bone density (predominantly diaphyseal) and aregenerative corticosteroidsensitive anemia. 